\title{
InGaN Super-Lattice Growth for Fabrication of Quantum Dot Containing Microdisks
}

\author{
H.A.R. El-Ella* a, F. Rol b, D. P. Collins ${ }^{\text {c }}$, M.J. Kappers a, R.A. Taylor ${ }^{\text {c }}$, E.L. Hu ${ }^{\text {b }}$, R.A. Oliver ${ }^{a}$ \\ * Corresponding author Tel.: +44 1223334368 \\ E-mail address: he234@,cam.ac.uk (H.A.R. El-Ella) \\ a Department of Materials Science and Metallurgy, University of Cambridge, Pembroke Street, Cambridge CB2 3QZ, UK \\ ${ }^{b}$ School of Engineering and Applied Sciences, Harvard University, Oxford Street, Cambridge MA 02138, USA \\ ${ }^{c}$ Clarendon Laboratory, Department of Physics, University of Oxford, Parks Road, Oxford OX1 3PU, UK
}

\begin{abstract}
Microstructural characterisation of a heterostructure containing an $\operatorname{In}_{\mathrm{x}} \mathrm{Ga}_{1-\mathrm{x}} \mathrm{N} / \mathrm{In}_{\mathrm{y}} \mathrm{Ga} \mathrm{1}_{-\mathrm{y}} \mathrm{N}$ super-lattice sacrificial layer (SSL), an AlGaN etch stop layer and an InGaN quantum dot layer has been carried out. These structures are intended for photoelectrochemical-etch mediated fabrication of undercut microdisks and were found to generate addition dislocations due to the additional strain energy imposed by the growth and inclusion of the InGaN quantum dot layer. Micro-photoluminescence was also carried out and showed the unexpected formation of quantum dots within the SSL. An equilibrium critical stack thickness model corresponding to the total thickness of the heterostructure that would favour the additional generation of dislocations was formulated through an energy-balance consideration. This correlated will well with the experimental results and a prediction of the SSL indium composition for the full structure that would not lead to additional dislocation generation could thus be made.
\end{abstract}

\section{Keywords}

Metalorganic Vapour Phase Epitaxy

Nitrides

Dislocations

InGaN Quantum Dots

Microdisks 


\section{Introduction}

Due to their exceptionally large exciton binding energies and oscillator strengths, group III nitrides are exciting candidates for the investigation of light-matter interactions. It has been predicted that achieving strong coupling between exciton states and photons within these systems will lead to exciton-polariton formation being attained and sustained at higher temperatures than have so far been achieved [1] [2]. Furthermore, it is hoped that this will lead to the observation of entangled states and quantum correlation at non-cryogenic temperatures [3]. Microdisks incorporating confined emitters such as quantum dots (QDs) are promising systems for such observations due to the inherent strong whispering gallery modes. Although strong optical coupling between QDs and microcavities has been demonstrated in various semiconductor systems, this has yet to be achieved within the nitrides. However, nitride-based undercut microdisks containing quantum wells have been successfully fabricated with high quality $(Q)$ factors and low threshold lasing has been demonstrated [4] [5] [6].

One approach to the fabrication of undercut microdisks from III-nitrides is through the use of a superlattice sacrificial layer (SSL) between an optically active membrane and the pseudo-substrate in conjunction with a selective photoelectrochemical etch (PECE). This process was developed by Haberer et al. [7] and further refined by Tamboli et al. [4] who suggested that due to the polarisation inherent within c-plane nitrides, the sacrificial layer needs to consist of a super-lattice in order to modulate the band edge and ensure a homogenous distribution of excited charge carriers across the layer during the etch process. The growth of such heterostructures is in itself a challenge, due to the accumulated strain between the heterostructure layers, and potential strain relaxation which can generate extra dislocations (in addition to those already present in the pseudo-substrate). These dislocations affect the local electronic and strain state, reducing the local etch rate [8] and creating non-radiative recombination centres [9]. The reduced etch rate around dislocations leads to the formation of 'whiskers' on surfaces and can significantly alter the target properties of micron-scale structures. It is therefore beneficial to design and grow heterostructures with suitable compositions and dimensions that will not relax and introduce additional dislocations.

In this article we investigate the origin and propagation of dislocations within a heterostructure design intended for PECE-mediated fabrication of microdisk cavities. Atomic force microscopy (AFM) and transmission electron microscopy (TEM) are used to investigate the occurrence of strain relaxation, and identify the origin of dislocations within the heterostructure. A model for the relaxation of the system, based on the energy-balance model first proposed by People \& Bean [10] and then further reformulated by Freund and Suresh [11] and Holec et al. [12] [13] will then be outlined in order to explain the onset of strain-induced relaxation within the heterostructure. 


\section{Experimental}

The complete structure intended for PECE-mediated fabrication of undercut microdisks is shown in Fig. 1. This was grown through metal-organic vapour phase epitaxy (MOVPE) and consists of an $\operatorname{In}_{x} \mathrm{Ga}_{1}$ ${ }_{x} \mathrm{~N} / \mathrm{In}_{\mathrm{y}} \mathrm{Ga}_{1-\mathrm{y}} \mathrm{N}$ SSL grown on an n-doped $\mathrm{GaN}$ pseudo-substrate, capped by a thin ( $\left.10 \mathrm{~nm}\right)$ layer of $\mathrm{GaN}$ followed by an $\mathrm{Al}_{0.19} \mathrm{Ga}_{0.81} \mathrm{~N}$ etch stop layer and a $\mathrm{GaN}$ membrane containing an InGaN QD layer. (The indium content of this QD layer is nominally $20 \%$, based on the growth approach used, but varies significantly through the layer). The design is intended for the creation of undercut microdisks where the QD-containing microdisk consists of all the layers above the SSL which serves as the post. A GaN layer between the AlGaN and SSL was included in order to protect the InGaN layers from the temperature ramp required prior to the AlGaN growth.

Six variants of the schematic in Fig.1 were also grown through MOVPE: two nominally identical structures labelled $\mathbf{A}$ and $\mathbf{B}$ with a slight difference in the SSL In composition, due to slight drift in the reactor temperature with time; three SSL structures without any capping layers and with varying SSL In compositions, labelled $\mathbf{C}, \mathbf{D}$ and $\mathbf{E}$; and an identical structure to $\mathbf{A}$ excluding the QD layer but subject to an identical growth temperature programme, labelled $\mathbf{F}$. The SSL indium compositions of all the structures are given in table 1. A control QD layer was also grown consisting of $\operatorname{In}_{0.2} \mathrm{Ga}_{0.8} \mathrm{~N}$ QDs with a $10 \mathrm{~nm}$ GaN cap grown on an n-doped GaN pseudo-substrate.

All samples were grown on $c$-plane $\mathrm{GaN}$ pseudo-substrates using sapphire substrates in a 6 x 2" Thomas Swan close-coupled showerhead reactor. (The pseudo-substrate growth method is described in more detail in [14]). Trimethylgallium, trimethylindium, trimethylaluminium and ammonia were used as precursors, with $\mathrm{N}_{2}$ used as a carrier gas for the growth of all indium-containing layers and $\mathrm{H}_{2}$ carrier gas for all other layers, except for the GaN layers grown between the SSL and the AlGaN layer and the first $10 \mathrm{~nm}$ of the $\mathrm{GaN}$ capping layer on top of the QDs. These two GaN layers were grown under $\mathrm{N}_{2}$ in order to protect the surface of the SSL and the QD layer from being exposed to and degraded by $\mathrm{H}_{2}$. The InGaN QD layer was grown using a self-assembled droplet epitaxy method which is described in more detail in [15].

All layer compositions were determined through x-ray diffraction (XRD) using the 2 curves of the (0004) reflection and the 2 reciprocal space map of the (1015) reflection. These reflections were measured in a Philips PW3050/65 High-Resolution diffractometer using $\mathrm{Cu} \mathrm{K}$ radiation. Layer thicknesses were estimated using a combination of in situ optical reflectivity and XRD measurements of fringe spacings of $50 \mathrm{~nm}$ calibration layers. Reference calibration layers were grown separately for XRD measurements. Defect densities were calculated from $1 \mu \mathrm{m} \times 1 \mu \mathrm{m}$ atomic force microscopy (AFM)

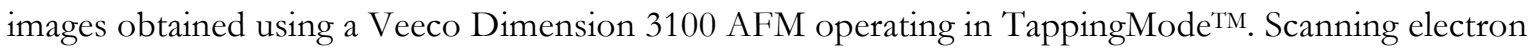
microscope (SEM) images were taken using a FEI XL-30s FEG SEM operating at $5 \mathrm{kV}$. Defect type and propagation through the heterostructures were probed with cross-sectional TEM using a Philips CM30 TEM operating at $300 \mathrm{kV}$. Cross-sectional TEM samples were prepared though wedge polishing followed by ion-beam thinning. Micro-photoluminescence $(\mu \mathrm{PL})$ was carried out at $4.5 \mathrm{~K}$. Luminescence was excited using a picosecond mode-locked Ti-sapphire laser emitting at $790 \mathrm{~nm}$ and focussed to a $2 \mu \mathrm{m}$ spot. (This two-photon excitation technique is explained elsewhere [16]). The emission from the samples was dispersed using a $0.3 \mathrm{~m}$ grating spectrometer with a Peltier cooled Si-based charge-coupled detector attached. 


\section{Results \& Discussion}

Initial $\mu \mathrm{PL}$ of structure $\mathbf{A}$ is shown in Fig. 2 along with the spectrum of the control QD layer taken under similar conditions. The spectrum for structure $\mathbf{A}$ shows a broad distribution of sharp peaks centred around $450 \mathrm{~nm}$ and extending down towards $420 \mathrm{~nm}$ confirming the formation and inclusion of InGaN QDs within the structure. The sharp cluster of peaks seen in the spectral window of 390-430 nm, in Figure 4(a) and absent in Fig. 4(b) arise from the SSL. The sharp nature of the emission lines seen in this wavelength range suggests unexpected formation of QD-like structures within the SSL. Fig. 3 shows a SEM image of a fabricated microdisk from sample $\mathbf{A}$, with protruding whiskers from the bottom surface of the microdisk visible. Cross sectional TEM images of the full structures $\mathbf{A}$ and $\mathbf{B}$ (pre-fabrication) are shown in Fig. 4. The figures show bright field images and weak-beam dark field images (taken around the $<10 \overline{1} 0>$ zone axis, with $g=[1 \overline{2} 10]$ to highlight the presence of edge and mixed type dislocations), showing misfit dislocations and threading edge-type/mixed dislocations originating in the GaN pseudosubstrate. Additional dislocation loops can be seen stemming from within the SSL resulting in an increased density of dislocations threading through the QD-containing membrane. The loops contain misfit segments with line vectors in the $\{0001\}$ plane, indicating dislocation generation due to strainrelaxation of the layers.

The TEM images suggest the generation of dislocations to achieve strain relaxation within the SSL. This effect was further quantified using AFM. Surface pit densities corresponding to threading dislocations were counted from $1 \mu \mathrm{m} \times 1 \mu \mathrm{m}$ AFM scans of samples $\mathbf{A}$ and $\mathbf{B}$, and compared to those found from a standard pseudo-substrate and the control QD layer. Fig. 5 shows $1 \mu \mathrm{m}$ surface scans of sample $\mathbf{A}$, the pseudo-substrate and the control QD sample, in which small pits associated with threading dislocations are observed. In Fig 5(a) and (c) some of the small pits associated with edge dislocations are arrowed, for clarity. The observed pit densities are summarised in table 1. Fig. 5(a) and 5(c) show terraces characteristic of high temperature $\mathrm{GaN}$ growth, whereas 5(b) shows spirals and step pinning resulting from the low temperature growth of the GaN cap. Application of a Student's t-test showed that the difference in calculated averages between the full structures $\mathbf{A}$ and $\mathbf{B}$, and the $\mathrm{GaN}$ pseudo-substrate/QD control samples were statistically significant within a certainty of $99.9995 \%$. No statistically significant difference was found between the GaN pseudo-substrate and the QD control sample. This suggested that there was no dislocation generation due to the QD layer alone and that a significant number of threading dislocations were being generated within the SSL, most likely due to plastic relaxation of the strained SSL layers.

To investigate the onset of strain-relaxation of the SSL layers, structures $\mathbf{C}, \mathbf{D}$ and $\mathbf{E}$ with decreasing indium compositions were grown and characterised in a similar manner. Their compositions and surface pit densities are summarised in table 1 and are similar to those of the pseudo-substrate/QD control layer. Fig. 6(a) and 6(b) show $1 \mu \mathrm{m} \times 1 \mu \mathrm{m}$ AFM surface scans of structure $\mathbf{C}$ and $\mathbf{E}$ displaying characteristic patchy hillocks and both small and large hexagonal v-pits associated with the threading dislocations. As well as potentially affecting the reflectivity of the undercut surfaces on the microdisks, the surface morphology may also limit the ability to detect smaller pits associated with edge-type dislocations resulting from any plastic relaxation. However, misfit and threading edge-type dislocations from within the SSL were also not observed in TEM examination of either sample $\mathbf{C}$ or $\mathbf{E}$, unlike in samples $\mathbf{A}$ and $\mathbf{B}$. Since samples B and C have the same composition of the SSL layers, this shows that without the overgrowth of the upper layers of the full structure, strain relaxation of the SSL at this composition does not occur. Strain relaxation during the growth of the upper layers could occur for two reasons: (a) if enough strain energy exists within the SSL to make strain relaxation favourable, the thermal activation energy for dislocation loops to glide in from the surface may be provided by the higher temperatures reached during the growth of the upper layers, or (b) if insufficient strain energy exists within the SSL to 
make strain relaxation favourable, the growth of the InGaN QD layer (but not the AlGaN layer as it is under tensile strain which compensates the compressive strain in the InGaN SSL) may increase the overall strain energy within the whole structure to a point where relaxation is favourable. To assess the feasibility of these two mechanisms an identical structure to $\mathbf{A}$ was grown, structure $\mathbf{F}$ which excludes the QD layer but was subject to an identical schedule of growth temperatures.

Using AFM, the surface pit density of structure $\mathbf{F}$ was assessed and a Student's t-test was then used to compare the average dislocation density in this sample with that of a GaN pseudo-substrate. No statistically significant difference was found, suggesting that no additional dislocations were being generated within this structure. As structure $\mathbf{F}$ was grown with an identical temperature program to that of structures $\mathbf{A}$ and $\mathbf{B}$, one of the two dislocation generation mechanisms mentioned above can be ruled out: dislocations are not forming in the SSL during the growth of the upper layers solely because the high temperature growth provides the required activation energy for the dislocation loops to glide in from the surface. The absence of strain relaxation in structure $\mathbf{F}$ therefore suggests that in structures $\mathbf{A}$ and $\mathbf{B}$ the combination of the indium contents of the QD layer and the SSL layers resulted in a critical amount of strain energy making the introduction of misfit dislocations energetically favourable. This phenomenon may be described as relaxation following an exceeding of the critical stack thickness. This suggestion will be theoretically assessed in Section 4.

Fig. 7 shows two $\mu$ PL spectra from sample F; 7(a) shows a typical spectrum and 7(b) a more unusual one. Neither spectrum shows the QD emission band seen in Fig. 2 (as expected since the QD layer is absent). Emission from the SSL is again seen at wavelengths from $395-420 \mathrm{~nm}$, and as in Fig 2, unexpected sharp resolution-limited peaks appear in this spectral window as can be seen in Fig 7(b), suggesting the existence of QD-like structures within the SSL. This is unexpected and rarely encountered in structure $\mathbf{F}$ in comparison with structure $\mathbf{A}$ where sharp peaks are almost always seen in this spectral window. This suggests that strongly confined emitters (or QDs) are occasionally formed within the SSL in sample F, but are far more frequent in sample $\mathbf{A}$. The possible mechanism resulting in the formation of strongly confined emitters in the SSL has yet to be determined, but one possibility is that they form via spinodal decomposition within relaxed material. (Both experimental and theoretical studies have previously suggested that spinodal decomposition is suppressed in biaxially strained material, but possible in relaxed material [17]). Since structure A exhibits significant misfit dislocation formation (and hence strain relaxation), and structure F does not, this would explain why QD-like features are seen more frequently in the SSL in $\mathbf{A}$ than in $\mathbf{F}$. In the case of structure $\mathbf{F}$, some strain relaxation may occur around the v-pits of the SSL layer during growth. so that occasional QD-like features are observed. 


\section{Critical Stack Thickness Modelling}

Using a simple and intuitive energy balance model, we can equate the total strain energy density of a structure to the energy of a dislocation required to relieve that strain, and define a critical stack thickness (CST) when they are equal - the point where it becomes energetically favourable for the structure to introduce dislocations. Here, the CST is defined as the total accumulated thickness of a given psuedomorphic multi-layer heterostructure and is modelled as a function of the alloy composition of a specific layer or set of layers, given a heterostructure with a constant number of layers $k$ and a constant thickness ratio between them. A heterostructure can be approximated as a single layer through defining an average isotropic areal strain energy density with a total thickness of $\mathcal{J}_{S}$ defined as:

$\bar{\varepsilon}_{S}=2 \bar{\mu}\left(\frac{1+\bar{v}}{1-\bar{v}}\right)\left(\frac{\sum_{k=1}^{n}\left(\epsilon_{k}\right)^{2} l_{k}}{\sum_{k=1}^{n} l_{k}}\right) \cdot \mathcal{T}_{S}$

where $l_{k}$ is the thickness of the $k^{\text {th }}$ layer, $\bar{\mu}$ and $\bar{v}$ are the shear modulus and Poisson ratio of the averaged layer, respectively, and $\epsilon_{k}$ is the strain in the $k^{\text {th }}$ layer. Assuming pseudomorphism, the strain within two individual layers $k_{1}$ and $k_{2}$ are related and the strain in one layer given as [18]:

$\epsilon_{k_{1}}=\frac{a_{k_{2}}}{a_{k_{1}}} \epsilon_{k_{2}}+\frac{a_{k_{2}}-a_{k_{1}}}{a_{k_{1}}}$

where $a_{k}$ is the in-plane lattice constant of the strained layer $k$. We can then equate the energy of a dislocation occurring in a specific layer/ group of identical layers to that of the areal strain energy density of the whole heterostructure. The CST is therefore interpreted as the point when these two energies are equal, i.e. when all the strain energy is relieved by the introduction of mixed dislocations within a specific layer/group of layers within the heterostructure (not the whole structure). Using the energy of a mixed dislocation in a linear isotropic continuum, the following expression can be formulated for the CST:

$\mathcal{T}_{S}=\frac{\mu b^{2}\left(1-v \cos ^{2} \theta\right)}{4 \pi(1-v) \bar{\varepsilon}_{S}} \ln \left(\frac{\mathcal{T}_{S}}{b}\right)$

where $b$ is the Burgers vector of the dislocation, $\theta$ is the angle between the Burgers vector and the dislocation line. A Lambert $W$ function is used to solve this equation analytically (more details can be found in [19] and [20]), and Vegards law is used to derive all alloy parameters.

The CST for the prismatic slip system $\frac{1}{3}\langle 11 \overline{2} 3\rangle\{1 \overline{1} 01\}$ is calculated for structures $\mathbf{A}, \mathbf{D}$ and $\mathbf{F}$ as a function of the In composition in the second layer of the SSL and shown in Fig 8. The strain energy of the QD layer is independently approximated and added as a layer strain contribution to the accumulated strain energy rather than averaged with the other layers. Although its strain is inextricably linked, this is done because it is not a continuous layer and therefore is not biaxially strained in relation to the rest of the layers. Bearing in mind these approximations, Fig. 8 shows that the QD layer in the full structure drastically increases the overall strain energy density (as would be expected) and results in a $\sim 40 \%$ lower CST value then the corresponding structure that excludes the growth of a QD layer. Fig. 8 also plots the actual total thickness of structures $\mathbf{A}, \mathbf{D}$ and $\mathbf{F}$ in order to show their relative proximity to the theoretical CST. The thickness of structures $\mathbf{D}$ and $\mathbf{F}$ are well below their theoretical CST values and therefore relaxation would not be expected, whereas the thickness of structure $\mathbf{A}$ is just above its theoretical CST value and therefore relaxation is expected. The model is therefore consistent with the experimental data even when taking into account the associated error in both the measured thickness and indium composition of the second SSL layers. 
This analysis can be taken a step further to predict the ideal composition of the SSL layers for the full structure $\mathbf{A}$, the ideal structure being a structure that contains as much indium as possible (to facilitate PECE fabrication) without introducing dislocations. Fig. 9 shows a contour graph of the CST for structure $\mathbf{A}$ as a function of the indium composition in both layers of the SSL $\left(\operatorname{In}_{\mathrm{x}} \mathrm{Ga}_{1-\mathrm{x}} \mathrm{N} / \mathrm{In}_{\mathrm{y}} \mathrm{Ga}_{1-\mathrm{y}} \mathrm{N}\right)$, labelled $\mathbf{x}$ for the first layer and $\mathbf{y}$ for the second layer. From Fig. 9 we can see that the theoretical CST exceeds the measured thickness of $\mathbf{A}(\sim 290 \mathrm{~nm})$ with any $\mathbf{x} / \mathbf{y}$ combination where $\mathbf{x} \leq 4 \%$ and $\mathbf{y} \leq 6 \%$. The CST stays well above $\sim 290 \mathrm{~nm}$ up to an $\mathbf{x} / \mathbf{y}$ combination of $\sim 6 \% / 9 \%$ provided that the difference between these two values does not exceed $\sim 3 \%$. In order to achieve a pronounced band edge oscillation necessary for PECE, it would be necessary to use two compositions with the highest difference within these values as well as to the surrounding GaN. Although the formulated CST model correlates well with the experimental findings, the model has a low error tolerance, especially for intricate and highly strained heterostrutures, for which the CST may change drastically for small increments in the composition parameters that are smaller than the inherent experimental errors. Taking this into account, an optimal SSL indium content would therefore be around $\sim 5 \% / 8 \%$ for structure A. In any case, it is important to note that this model is an equilibrium model, and does not consider dislocation sources or mechanisms. Above the CST, all dislocations are assumed to appear together suddenly, and their energy will fully replace that of the strain. Finally, although the misfit dislocations introduced are most likely edge dislocations, a mixed dislocation energy is used to account for the possible occurrence of both, which will introduce further errors. Nonetheless the model suggests that the experimental results are well accounted for by an exceeding of the CST. 


\section{Conclusion}

In an SSL-containing heterostructure intended for PECE-mediated fabrication dislocation loops were observed due to relaxation within the SSL layers. Subsequent growth of uncapped SSL layers with equal and varying compositions was shown not to generate additional dislocations, showing that the SSL layers alone do not lead to relaxation. Growth of a structure excluding the QD layer but subjected to the same temperature program as the full structure showed that the dislocation formation only occurred in the presence of the QD layer. This pointed towards the CST being reduced by the contribution of the strain energy of the QD layer to the overall strain energy density of the full structure. An equilibrium CST model was derived through an energy-balance formulation, and corresponded well with the experimental results taking into account the associated errors. From this model, a prediction of an optimal SSL indium content for the full structure that will not lead to strain-relaxation was given. The unexpected formation of strongly confined emitters within the $\operatorname{In}_{x} \mathrm{Ga}_{1-\mathrm{x}} \mathrm{N} / \mathrm{In}_{\mathrm{y}} \mathrm{Ga}_{1-\mathrm{y}} \mathrm{N}$ SSL was also shown.

\section{Acknowledgments}

This work was supported in part by the EPSRC. RAO would like to acknowledge funding from the Royal Society. The author would like to thank Dr Yucheng Zhang and Dr Tom Sadler for useful discussions and help with TEM characterisation. 


\section{References}

[1] J. Kasprzak, M. Richard, S. Kundermann, A. Baas, P. Jeambrun, J. M. J. Keeling, F. M. Marchetti, M. H. Szyma ska, R. André, J. L. Staehli, V. Savona, P. B. Littlewood, B. Deveaud, Le Si Dang, Nature 443 (2006) 409.

[2] S. Christopoulos, G. Baldassarri Höger von Högersthal, A. J. D. Grundy, P. G. Lagoudakis, A.V. Kavokin, J. J. Baumberg, G. Christmann, R. Butté, E. Feltin, J.-F. Carlin, N. Grandjean, Phys. Rev. Lett. 98 (2007) 126405

[3] H. Deng, H. Haug, Y. Yamamoto, Rev. Mod. Phys. 82 (2010) 1489

[4] A.C. Tamboli, E.D. Haberer, R. Sharma, K.H. Lee, S. Nakamura, E.L. Hu, Nature Photonics 1 (2007) 61

[5] E.D. Haberer, Phys. Stat. Sol. C 2 (2005) 2845.

[6] D. Simeonov, E. Feltin, A. Altoukhov, A. Castiglia, J.-F. Carlin, R. Butté, N. Grandjean Appl. Phys Lett 92 (2008) 171102

[7] E.D. Haberer, R. Sharma, C. Meier, A.R. Stonas, S. Nakamura, S.P. DenBaars, E.L. Hu, Appl. Phys. Lett. 85 (2004) 5179

[8] C. Youtsey, L.T. Romano, I. Adesida, Appl. Phys. Lett. 73 (1998) 797.

[9] J.S. Speck, S.J. Rosner Physica B 273 (1999) 24

[10] R. People, J.C. Bean, Appl Phys Lett 47 (1985) 322

[11] L. B. Freund and S. Suresh, Thin Film Materials: Stress, Defect Formation, and Surface Evolution, Cambridge University Press, Cambridge, 2003.

[12] D. Holec, Y. Zhang, D.V. Sridhara Rao, M.J. Kappers, C. McAleese, C.J. Humphreys J Appl Phys 104 (2008) 123514

[13] D. Holec, P.M. F. J. Costa, M. J. Kappers, C. J. Humphreys, J. Cryst. Growth 303 (2007) 314.

[14] R.A. Oliver, M.J. Kappers, J. Sumner, R. Datta, C.J. Humphreys, J. Cryst. Growth. 289 (2006) 506

[15] R.A. Oliver, G.A.D. Briggs, M.J. Kappers. C.J. Humphreys, S. Yasin, J.H. Rice, J.D Smith, R.A. Taylor Appl. Phys. Lett., 83 (2003) 755

[16] A.F. Jarjour, A.M. Green, T.J. Parker, R.A. Taylor, R.A. Oliver, G.A.D. Briggs, M.J.Kappers, C.J. Humphreys, R.W. Martin, I.M. Watson, Physica E 32 (2006) 119

[17] R.A. Oliver, S.E. Bennett, T. Zhu, D.J. Beesley, M.J. Kappers, D.W. Saxey, A. Cerezo, C.J. Humphreys, J. Phys. D: Appl. Phys 43 (2010) 354003

[18] N.J. Ekins-Daukes, K. Kawaguchi, J. Zhang Cryst Growth Design 2 (2002) 287

[19] A. Braun, K.M. Briggs, P. Böni, J Cryst Growth 241 (2002) 231

[20] C.H. Belgacem, M. Fnaiech, J Electron Mater (2010) DOI: 10.1007/s11664-010-1290-5

[21] WSxM C; www.nanotec.es 


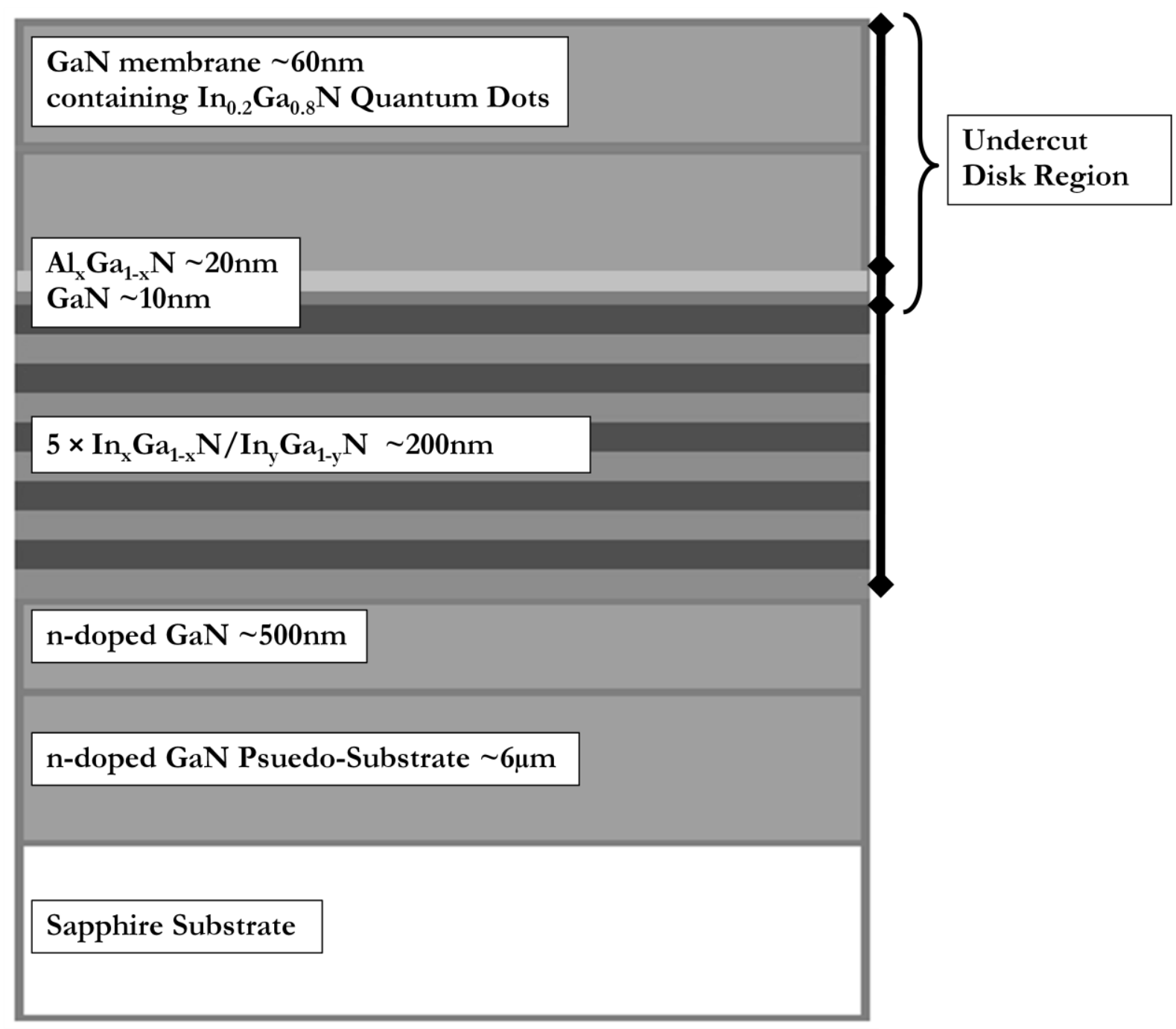

Figure 1 Schematic of structures A and B. All other structures grown for this study are modifications of this design. 

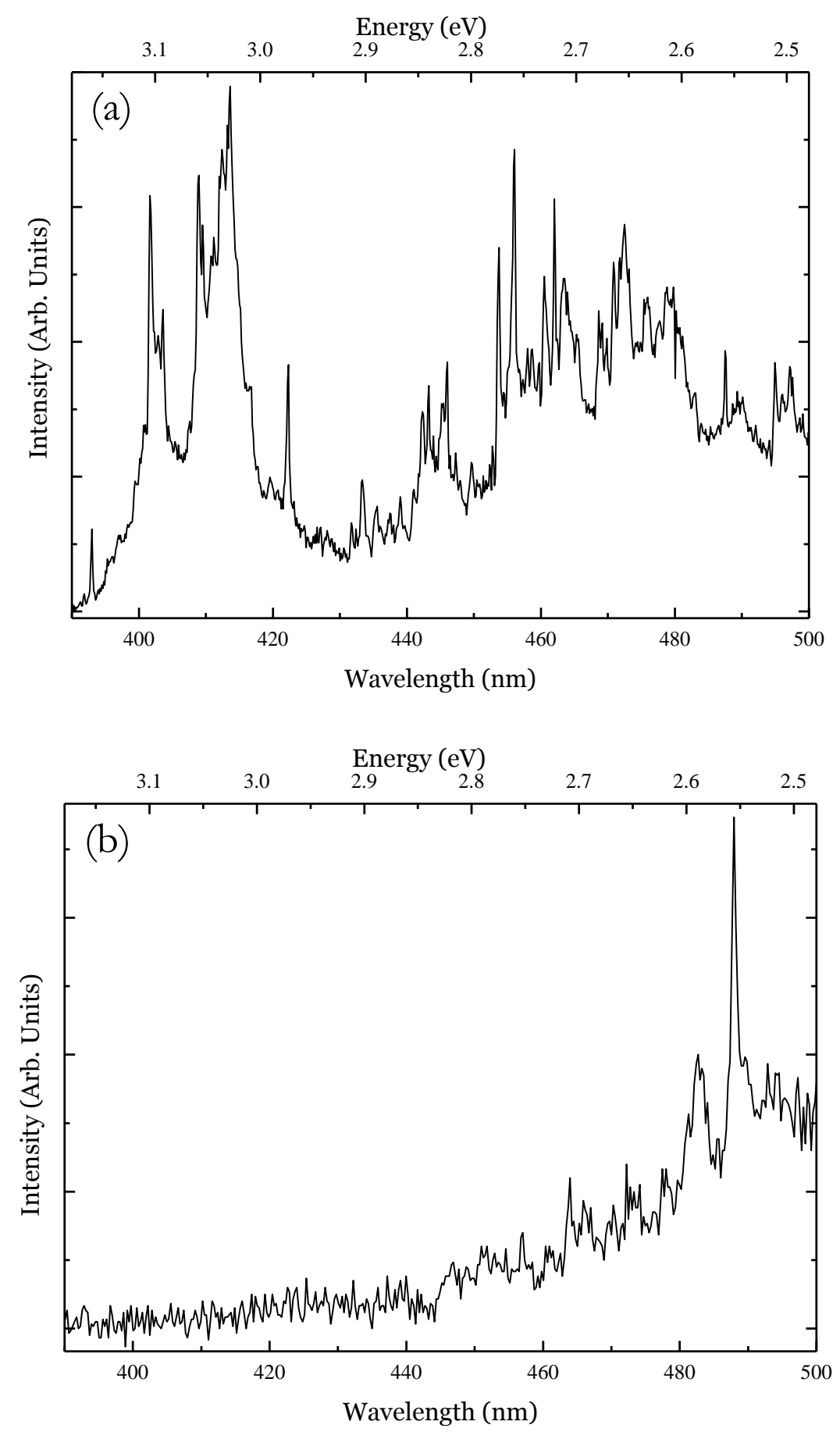

Figure 2 uPL spectra taken at $4.5 \mathrm{~K}$ of (a) structure A, and (b) QD control layer 


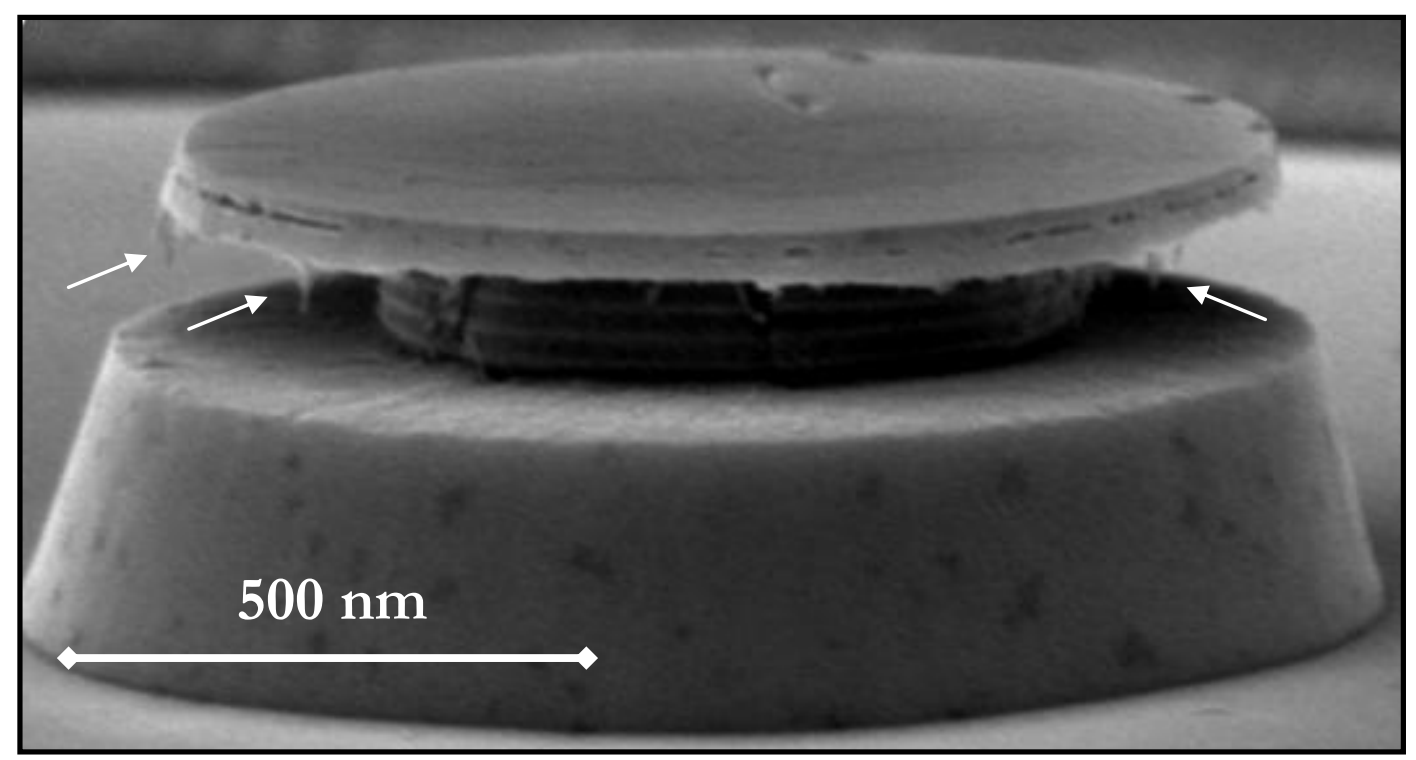

Figure 3 Scanning electron image of a $1 \mu \mathrm{m}$ disk created from structure A employing a PEC etch. Whiskers are labelled with arrows can be seen protruding from the edges and the underside of the disk 

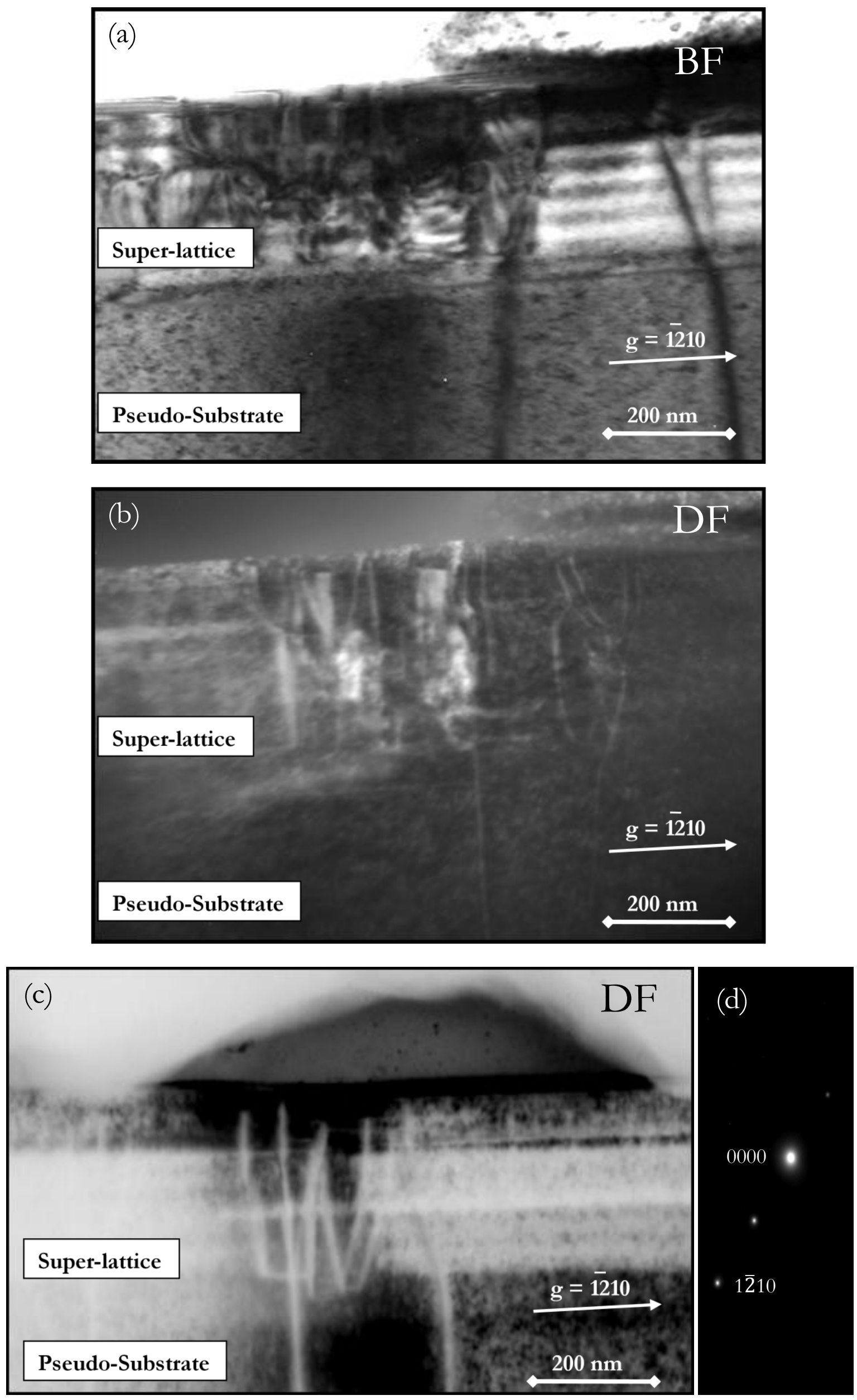

Figure 4 (a), (b) Bright field and weak-beam dark field images of structure A taken around the $<10 \overline{1} 0>$ zone axis, with $\boldsymbol{g}=[1 \overline{2} 10]$ for the dark-field images at $300 \mathrm{kV}$. (c) Weak beam dark field image of structure B taken using the same diffraction conditions. (d) Selected area diffraction pattern showing the diffraction condition. 


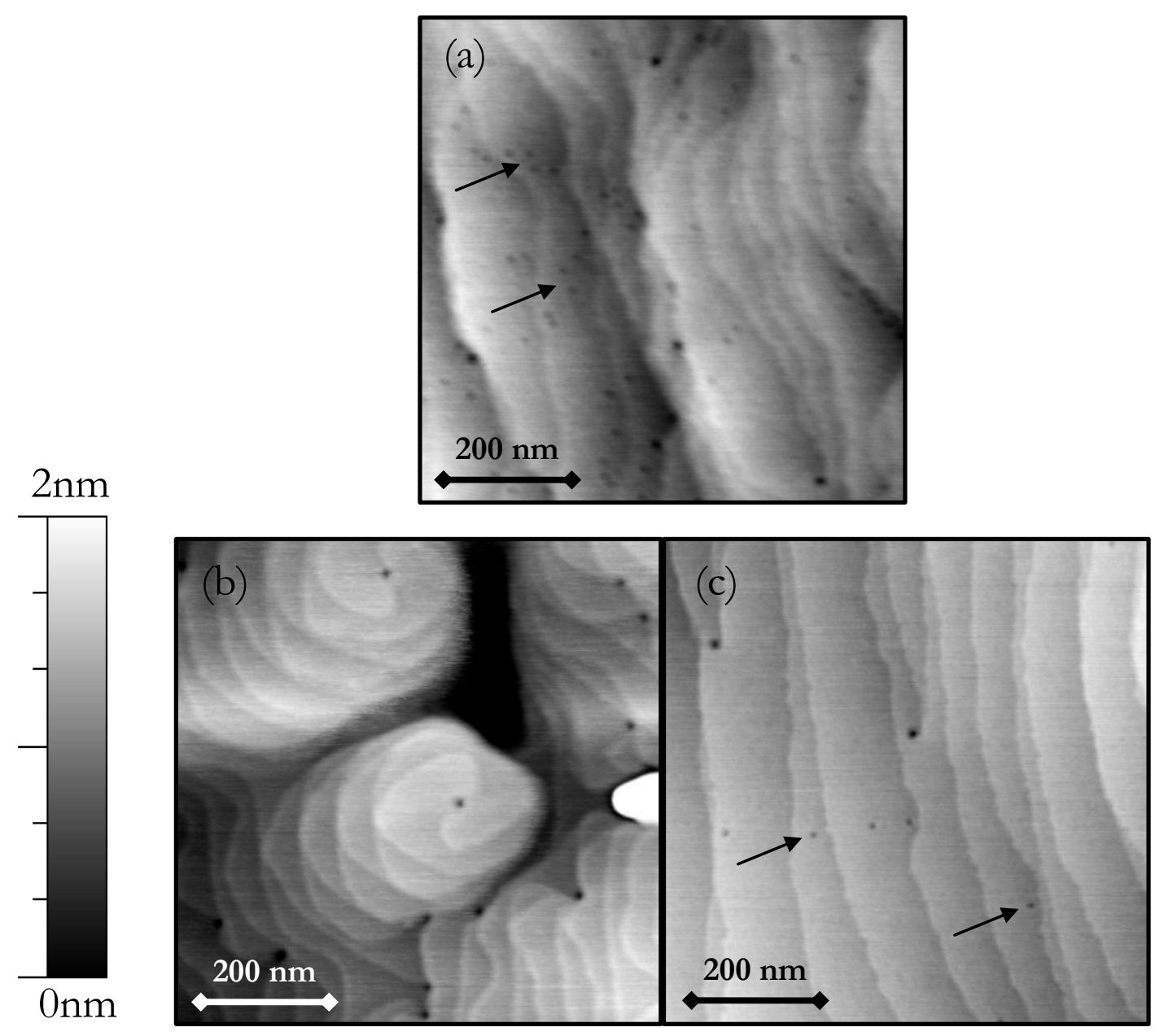

Figure $51 \mu \mathrm{m} \times 1 \mu \mathrm{m}$ AFM scans of (a) full structure A, (b) InGaN QD control layer (c) GaN pseudo substrate. In (a) and (c) arrows indicate small pits relating to edge dislocations.
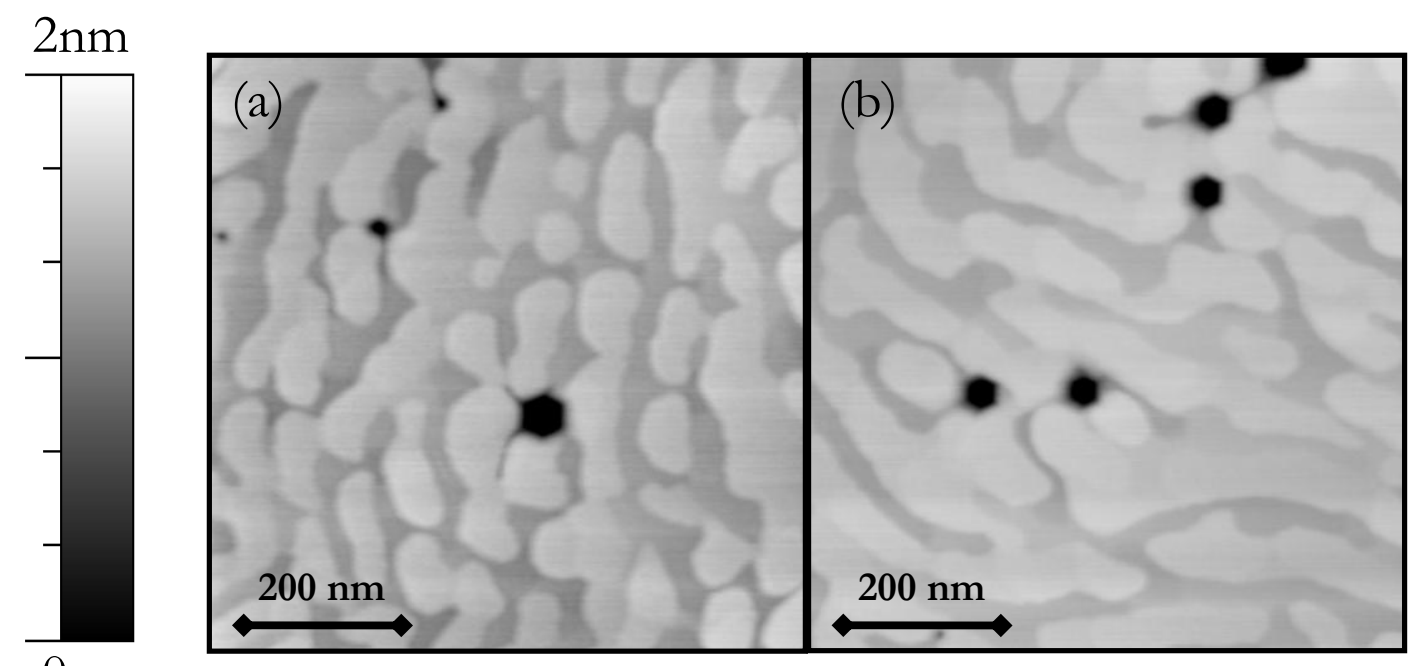

Onm

Figure $61 \mu \mathrm{m} \times 1 \mu \mathrm{m}$ AFM scans of $\operatorname{In}_{x} G a_{1-x} N / \operatorname{In}_{y} G a_{1-y} N$ SSL (a) structure $C$ and (b) structure $\mathrm{E}$ 

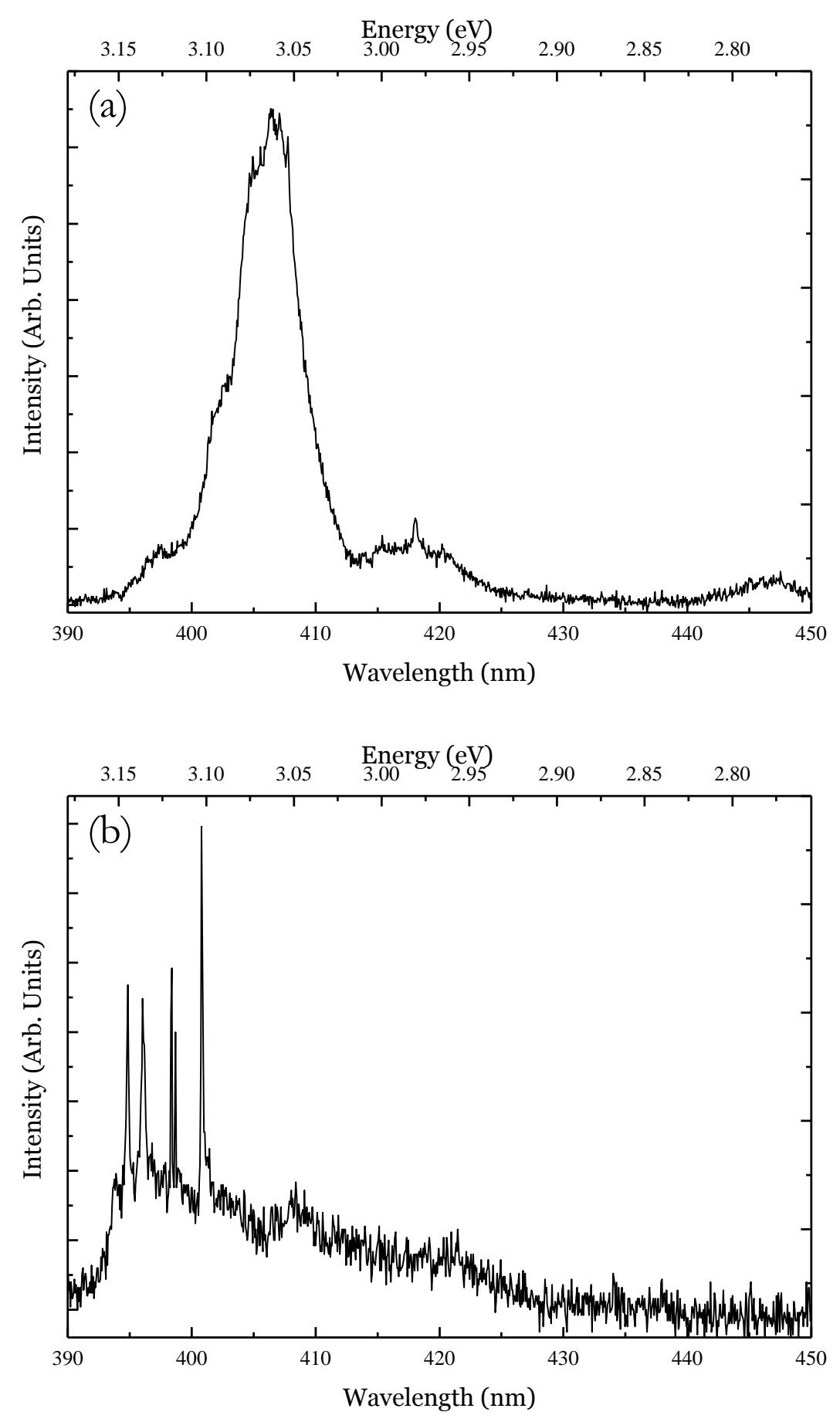

Figure $7 \mu \mathrm{PL}$ spectra of structure $\mathrm{F}$ taken at $4.5 \mathrm{~K}$ showing (a) typical spectrum and (b) a more unusual spectrum influenced by strongly confined emission. 


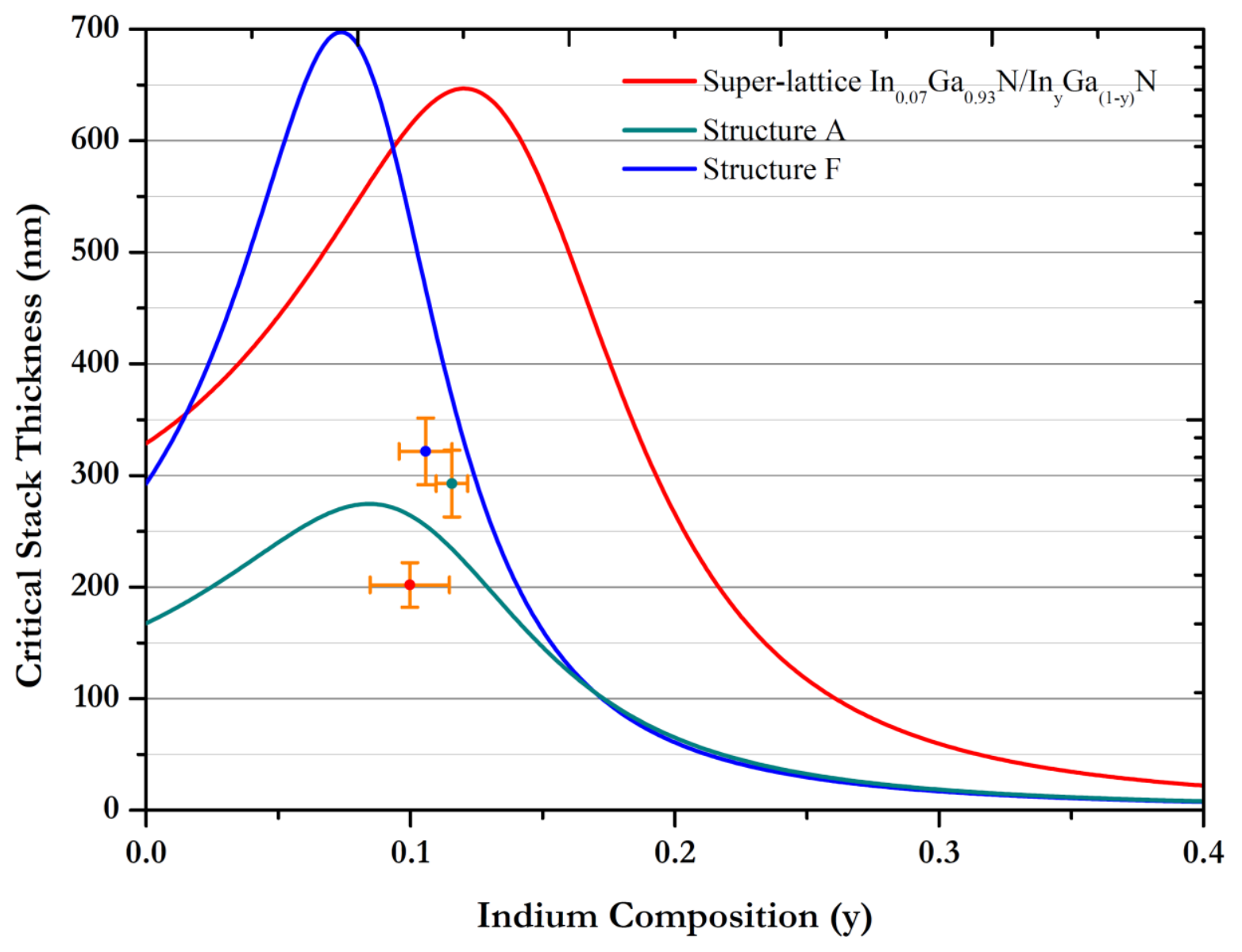

Figure 8 Theoretical variation in the CST with the indium content of the second SSL layer (y) for theoretical structures based on samples A, F and C, all with a first SSL layer composition of $7 \%$ indium. Points are also plotted (colour coded) for the experimentally determined composition and total stack thickness of the actual samples $\mathrm{A}, \mathrm{F}$ and $\mathrm{C}$. 


\section{Critical Stack Thickness (nm)}

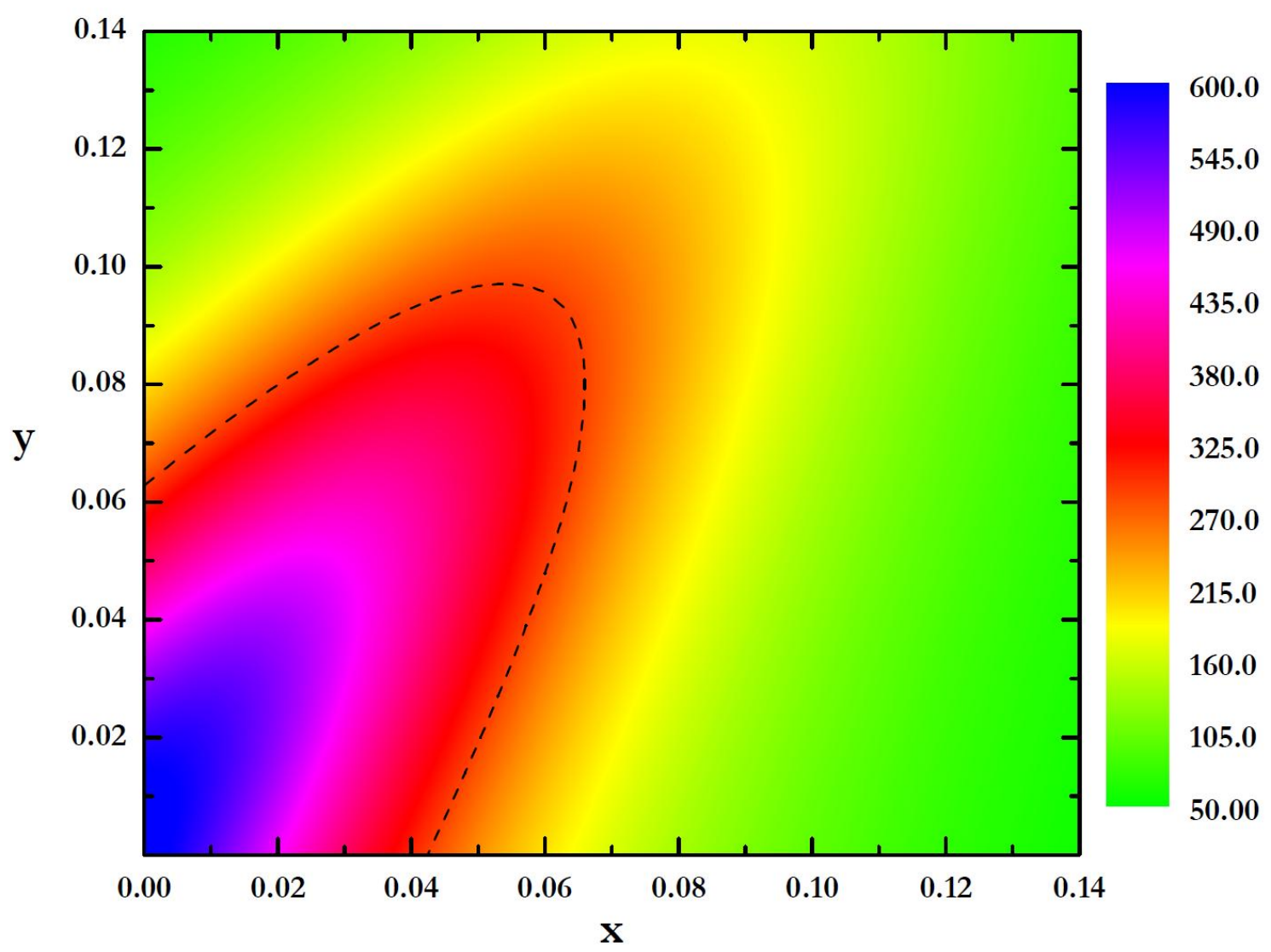

Figure 9 Contour map showing the theoretical variation in the CST for the structure shown in Figure 1 as a function of both compositions of the $\operatorname{In}_{x} G a_{1-x} N / \operatorname{In}_{y} G a_{1-y} N$ SSL. The experimentally determined thickness of $\mathrm{A}$ is traced with a dashed line. 


\begin{tabular}{cccc}
\hline Structure & $\begin{array}{c}\text { In \% in } \\
\text { SSL }\end{array}$ & Surface Pit Density $\left(\mathbf{c m}^{-2}\right)$ & $\begin{array}{c}\text { Total Thickness } \\
(\mathbf{n m})\end{array}$ \\
\hline A & $11 \% / 7 \%$ & $\sim(4 \pm 0.7) \times 10^{9}$ & $\sim 300$ \\
B & $10 \% / 7 \%$ & $\sim(3 \pm 0.5) \times 10^{9}$ & $\sim 290$ \\
C & $10 \% / 7 \%$ & $\sim(4 \pm 1.0) \times 10^{8}$ & $\sim 200$ \\
D & $9 \% / 6 \%$ & $\sim(4 \pm 1.0) \times 10^{8}$ & $\sim 200$ \\
E & $7 \% / 5 \%$ & $\sim(5 \pm 0.8) \times 10^{8}$ & $\sim 200$ \\
F & $11 \% / 7 \%$ & $\sim(6 \pm 0.7) \times 10^{8}$ & $\sim 300$ \\
$\begin{array}{c}\text { Pseudo- } \\
\text { Substrate }\end{array}$ \\
$\begin{array}{c}\text { QD } \\
\text { Control } \\
\text { Layer }\end{array}$ & - & $\sim(8 \pm 1.0) \times 10^{8}$ & - \\
\hline
\end{tabular}

Table 1 Summary of compositions, pit densities and total thickness of heterostructure excluding the GaN pseudo-substrate of all structures grown for this study. 